\title{
Free vibration analysis of non-symmetric FGM sandwich square plate resting on elastic foundations
}

\author{
H. Saidi, W. AddaBedia, A. Fekrar, F. Ismail Salman, A. Tounsi
}

Laboratoire des Matériaux et Hydrologie, Université de Sidi Bel Abbes, Département de Génie Civil, Faculté de Technologie, Algérie

\begin{abstract}
In this study, free vibration analysis of simply supported sandwich plate resting on elastic foundation is examined. In this model, the displacements vary as a sinusoidal function across the plate thickness and satisfy zero shear stress condition at the top and bottom surfaces of the plate. The governing equations are derived by employing the principle of Hamilton, These equations are solved via Navier type and the dynamic results are presented by solving eigenvalue problems. The numerical results obtained through the present analysis for free vibration are presented and compared with those available in the literature.
\end{abstract}

\section{Introduction}

Laminated composite plates are widely used in the aerospace, marine, construction industries, medical devices and many other areas [1-4] because of advantageous features such as high ratio of stiffness and strength to weight and low maintenance cost.

Functionally graded material (FGM) was proposed in 1984 [5]. The FGM is a composite material whose composition varies according to a performance required. It can be produced by a continuous variation of volume fractions of its constituents, which leads to continuously varying material properties of the FGM. This is the main difference between such a material and a usual laminated composite.

There have been a considerable number of studies on the plates resting on elastic foundations. In some of the analyses, a single parameter $\mathrm{k}_{0}$ is used to describe the foundation behavior [6]. In this method it is assumed that there is a proportional interaction between pressure and deflection of an applied point in foundation. This foundation is modeled by discrete vertical springs and does not take into account the transverse shear deformations in foundation (linear Winkler model). Some researchers have modeled the foundation with two different parameters. One of these models is the Pasternak type foundation model. The two parameter model of Pasternak takes into account the effect of shear interaction among the points in the foundation [7-10]. If the second parameter vanishes, the Pasternak foundation is reduced to the Winkler type.

This paper presents a sinusoidal shear deformation theory of plates for analyzing the free vibration response of functionally graded plates resting on WinklerPasternak elastic foundation. Numerical results are presented for simply supported square sandwich plate, the effect of foundation stiffness and the gradient index on the free vibration of FGM sandwich plate are also investigated.

\section{Theoretical formulations}

Let us consider a square sandwich plate of length " $a$ " and uniform total thickness " $h$ ", resting on Winkler-Pasternak elastic foundation, with Winkler stiffness " $\mathrm{K}_{0}$ " and shear stiffness " $\mathrm{K}_{1}$ ", as shown in Figure 1.

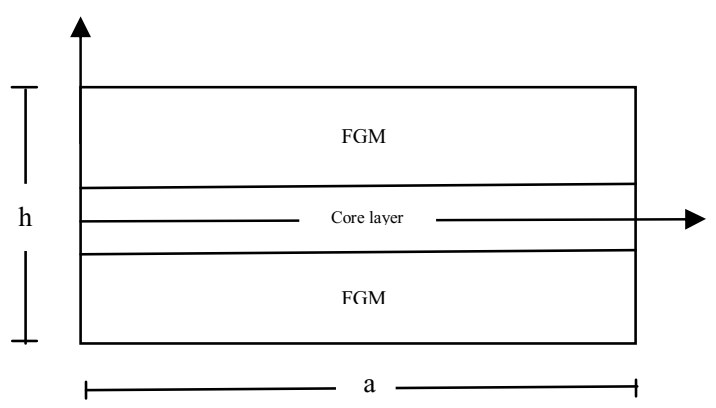

Figure 1. Schematic of FGM sandwich plate

The effective material for the faces, like Young modulus E, Poison's ration $v$ and mass density $\rho$, can be expressed as: 


$$
P^{(n)}(z)=P_{b}+\left(P_{t}-P_{b}\right) V^{(n)}
$$

where $P_{t}$ and $P_{b}$ denote the properties of the top and the bottom surfaces of layer 1 (bottom face), respectively, and vice versa for layer 3 (top face) depending on the volume fraction $\mathrm{V}^{(\mathrm{n})}(\mathrm{n}=1,2,3)$. The sandwich plate is symmetrical with respect to the mid-plane $\mathrm{z}=0$. The volume fraction $\boldsymbol{V}^{(\boldsymbol{n})}$ through the thickness of the sandwich plate faces follows a simple power-law while it equals unity in the core layer.

The displacement field is defined in the following equations:

$$
\begin{gathered}
u=u_{0}(x, y)-z w_{, x}+f(z) \theta_{x} \\
v=v_{0}(x, y)-z w_{, y}+f(z) \theta_{y} \\
w=w_{0}(x, y)
\end{gathered}
$$

where, $u, v, w$ are displacements in the $x, y, z$ directions, $u_{0}, v_{0}$, and $w_{0}$ are midplane displacements, $\theta_{x}$ and $\theta_{y}$ rotations of the $y z$ and $x z$ planes due to bending, respectively. $f(z)$ represents shape function determining the distribution of the transverse shear strains and stresses along the thickness.

The sinusoidal shear deformation theory (SSDT) of Touratier [11] is obtained by setting:

$$
f(z)=\frac{h}{\pi} \sin \left(\frac{\pi z}{h}\right)
$$

\section{Constitutive equations}

The constitutive equations for nth layer of laminated plate can be expressed as:

$$
\begin{aligned}
& \left\{\begin{array}{r}
x x \\
y y \\
\tau_{x y}
\end{array}\right\}=\left[\begin{array}{ccc}
\bar{Q}_{11} & \bar{Q}_{12} & 0 \\
\bar{Q}_{12} & \bar{Q}_{22} & 0 \\
0 & 0 & \bar{Q}_{66}
\end{array}\right]\left\{\begin{array}{l}
\varepsilon_{x x} \\
\varepsilon_{y y} \\
\gamma_{x y}
\end{array}\right\} \\
& \left\{\begin{array}{l}
\tau_{y z} \\
\tau_{z x}
\end{array}\right\}=\left[\begin{array}{cc}
\bar{Q}_{44} & 0 \\
0 & \bar{Q}_{55}
\end{array}\right]\left\{\begin{array}{l}
\gamma_{y z} \\
\gamma_{z x}
\end{array}\right\}
\end{aligned}
$$

where $\left({ }_{x x}, \sigma_{y y}, \tau_{x y}, \tau_{x z}, \tau_{z x}\right)$ and $\left(\varepsilon_{x x}, \varepsilon_{y y}, \gamma_{x y}\right.$, $\left.\gamma_{y z}, \gamma_{z x}\right)$ are the stress and strain components, respectively.

The normal and shear strain components are obtained as:

$$
\begin{gathered}
\varepsilon_{x x}=u_{0, x}-z w_{, x x}+f(z) \theta_{x, x} \\
\varepsilon_{y y}=v_{0, y}-z w_{, y y}+f(z) \theta_{y, y} \\
\gamma_{x y=} u_{0, y}+v_{0, x}-2 z w_{, x y}+f(z)\left(\theta_{x, y}+\theta_{y, x}\right)
\end{gathered}
$$

$$
\begin{aligned}
& \gamma_{y z}=\grave{f}(z) \theta_{y} \\
& \gamma_{x z}=\grave{f}(z) \theta_{x} \\
& \grave{f}(z)=\frac{d f(z)}{d z}
\end{aligned}
$$

\section{Governing equation}

Using Hamilton's energy principle derives the equation of motion of the FGM plate:

$$
\delta \int_{t 1}^{t 2}\left(U+U_{F}-K-W\right) d t=0
$$

where $U$ is the strain energy, $\mathrm{K}$ is the kinetic energy of the plate, $U_{F}$ is the strain energy of foundation and $\mathrm{W}$ is the work of external forces.

For Winkler-Pasternak foundation mode:

$$
f_{e}=K_{0} w+K_{1} \nabla^{2} w
$$

If the foundation is modeled as the linear Winkler foundation, the coefficient $K_{1}$ in (8) is zero.

\section{Numerical procedure}

The boundary conditions along the edges of the simply supported plate can be obtained as:

$$
\begin{aligned}
& N_{x}=v=w=M_{x}=P_{x}=\theta_{y}=0 \text { at } x=0, a \\
& N_{y}=u=w=M_{y}=P_{y}=\theta_{x}=0 \text { at } x=0, b
\end{aligned}
$$

For analytical solution of equations, the Navier method is used under the specified boundary conditions.

The displacement functions that satisfy the equations of boundary conditions are selected as the following Fourier series:

$$
\begin{aligned}
& u(x, y)=\sum_{m=1}^{\infty} \sum_{n=1}^{\infty} A_{m n} \cos \frac{m \pi x}{a} \sin \frac{n \pi y}{b} e^{i \omega t} \\
& v(x, y)=\sum_{m=1}^{\infty} \sum_{n=1}^{\infty} B_{m n} \sin \frac{m \pi x}{a} \cos \frac{n \pi y}{b} e^{i \omega t} \\
& w(x, y)=\sum_{m=1}^{\infty} \sum_{n=1}^{\infty} C_{m n} \sin \frac{m \pi x}{a} \sin \frac{n \pi y}{b} e^{i \omega t} \\
& \theta_{x}(x, y)=\sum_{m=1}^{\infty} \sum_{n=1}^{\infty} T_{x m n} \cos \frac{m \pi x}{a} \sin \frac{n \pi y}{b} e^{i \omega t} \\
& \theta_{y}(x, y)=\sum_{m=1}^{\infty} \sum_{n=1}^{\infty} T_{y m n} \sin \frac{m \pi x}{a} \sin \frac{n \pi y}{b} e^{i \omega t}
\end{aligned}
$$


where $A_{m n}, B_{m n}, C_{m n}, T_{x m n}, T_{y m n}$ are arbitrary parameters to be determined, and $\omega$ is the eigenfrequency associated with $(m, n)$ th eigen mode.

eigenvalue equation for any fixed value of $m$ and $n$, for free vibration problem:

$$
\begin{gathered}
\left([K]-\omega^{2}[M]\right)\{\Delta\}=\{0\} \\
{[K]=\left[\begin{array}{lllll}
a_{11} & a_{12} & a_{13} & a_{14} & a_{15} \\
a_{12} & a_{22} & a_{23} & a_{24} & a_{25} \\
a_{13} & a_{23} & a_{33} & a_{34} & a_{35} \\
a_{14} & a_{24} & a_{34} & a_{44} & a_{45} \\
a_{15} & a_{25} & a_{35} & a_{45} & a_{55}
\end{array}\right]} \\
{[M]=\left[\begin{array}{ccccc}
I_{1} & 0 & -\alpha I_{2} & I_{4} & 0 \\
0 & I_{1} & -\beta I_{2} & 0 & I_{2} \\
\alpha I_{2} & \beta I_{2} & -I_{3}\left(\alpha^{2}+\beta^{2}\right) & I_{5} & I_{5} \\
I_{4} & 0 & -\alpha I_{5} & I_{6} & 0 \\
0 & I_{4} & -\beta I_{5} & 0 & I_{6}
\end{array}\right]} \\
\{\Delta\}=\left\{\begin{array}{c}
A_{m n} \\
B_{m n} \\
C_{m n} \\
T_{x m n} \\
T_{y m n}
\end{array}\right\}
\end{gathered}
$$

For non trivial solutions of equation (10), the following determinant should be zero.

$$
\left|[K]-\omega^{2}[M]\right|=0
$$

\section{Numerical results and discussions}

Numerous examples are solved to ensure the accuracy of the present theory for the prediction of free vibration response, closed form solution are obtained using the Navier solution of simply supported FGM sandwich plates.

Table1. Material properties used in the FGM plate.

\begin{tabular}{|l|l|l|}
\hline Properties & Metal (Aluminium) & Ceramic \\
\hline $\mathrm{E}(\mathrm{GPa})$ & 70 & 380 \\
\hline$v$ & 0.3 & 0.3 \\
\hline$\rho\left(\mathrm{Kg} / \mathrm{m}^{3}\right)$ & 2707 & 3800 \\
\hline
\end{tabular}

As a first example, the natural frequencies of a nonsymmetric FGM square sandwich plate of type (2-2-1) $\left(\mathrm{P}=2, \mathrm{k}_{0}=10\right)$ for different values of Pasternak parameters are given in Figure 2, we can say that the fundamental frequencies increases as the Pasternak parameters increase.

Figure 3 present the fundamental frequencies of antisymmetric square sandwich plate of type $(2-2-1)(\mathrm{P}=2$, $\mathrm{k} 1=10$ ) for different values of Winkler parameters. It can be seen that the frequency increase gradually with increasing Winkler parameters.

Figure 4 and Figure 5 show the results of the natural frequencies of anti-symmetric FGM square sandwich plate of type (2-1-1), $(\mathrm{P}=2)$ for different values of Pasternak and Winkler parameters, respectively. It can be observed that the frequencies increase as the elastic foundation parameters increases.

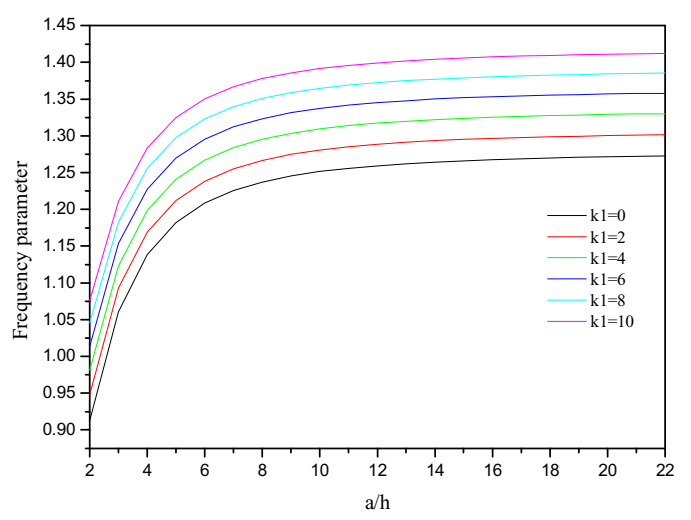

Figure 2. Fundamental frequency of side to thickness ratio of square FGM sandwich plate (2-2-1) for different values of Pasternak parameter $\mathrm{K}_{1}, \mathrm{~K}_{0}=10$

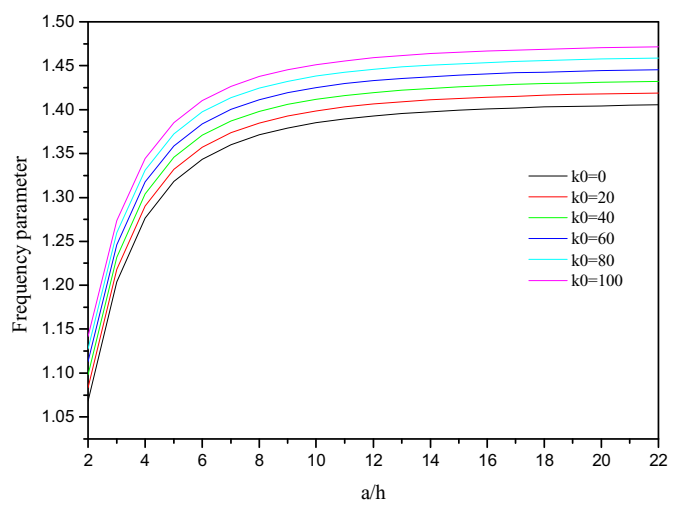

Figure 3. Fundamental frequency of side to thickness ratio of square FGM sandwich plate (2-2-1) for different values of Winkler parameter $\mathrm{K}_{0}, \mathrm{~K}_{1}=10$ 


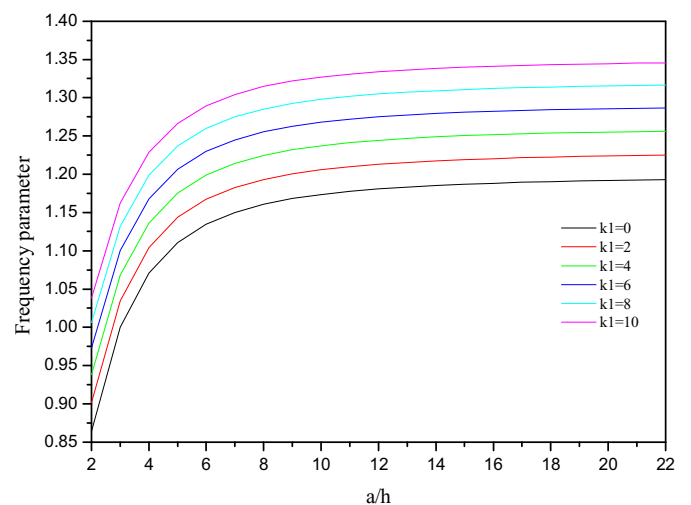

Figure 4. Fundamental frequency of side to thickness ratio of square FGM sandwich plate (2-1-1) for different values of Pasternak parameter $\mathrm{K}_{1}, \mathrm{~K}_{0}=10$

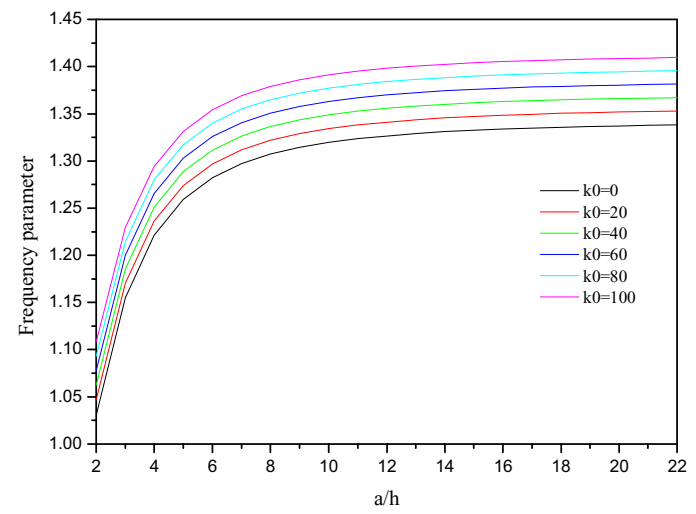

Figure 5. Fundamental frequency of side to thickness ratio of square FGM sandwich plate (2-1-1) for different values of Winkler parameter $\mathrm{K}_{0}, \mathrm{~K}_{1}=10$

\section{Conclusion}

In the present study, the free vibration analysis of antisymmetric FGM square sandwich plate resting on Winkler-Pasternak elastic foundation have been obtained on the assumption that the transverse shear displacement varies as a sinusoidal function across the plate thickness.

The equilibrium equations and the boundary conditions are derived using Hamilton's principle.

The effects of span to thickness ratio, and elastic foundation parameters on the fundamental frequencies are studied and it is observed that the natural frequencies increase as the side to thickness ratio increase.

It can be seen also from our results that the natural frequencies of the anti-symmetric FGM sandwich plate increase as the elastic foundation parameters increases.

\section{References}

1. SR. Swanson, Introduction to design and analysis with advanced composite materials. Prentice Hall Inc., (1997). 2. HG. Allen, Analysis and design of structural sandwich plates. Oxford: Pergamon Press., (1969).
3. RM. Jones, Mechanics of composite materials. Second ed. Taylor \& Francis, Inc., (1999).

4. AP. Mouritz, E. Gellert, P. Burchill, K. Challis, Review of advanced composite structures for naval ships and submarines. Compos Struct. 53, pp. 21-41, (2001) 5. M. Koizumi, FGM activities in Japan, Composites, 28, 14, (1997).

6. A. El Zafrany, S. Fadhil, and K. Al-Hosani, A New Fundamental Solution for Boundary Element Analysis of Thick Plates on Winkler Foundation, Int. J. Numer. Meth. Eng, 38, pp. 887-903, (1995).

7. MH. Omurtag and F. Kadioglu, Free Vibration Analysis of Orthotropic Plates Resting on Pasternak Foundation by Mixed Finite Element Formulation, Computers and Structures, 67, pp. 253-265, (1998).

8. AR. Setoodeh, and G. Karami, Static, Free Vibration and Buckling Analysis of Anisotropic Thick Laminated Composite Plates on Distributed and Point Elastic Supports using a 3-D Layer-wise FEM, Engineering Structures, 26, pp. 211-220, (2004).

9. Y. Xiang, S. Kitipornchai, and KM. Liew, Buckling and Vibration of Thick Laminates on Pasternak Foundation, J. Eng Mech. ASCE, 122, 1, pp. 54-63, (1996).

10. S. Hui-Shen, , JJ. Zheng, and XL. Huang, Dynamic Response of Shear Deformable Laminated Plates under Thermomechanical Loading and Resting on Elastic Foundations, Composite Structures, 60, pp. 57-66, (2003).

11. M. Touratier. An efficient standard plate theory, Int. J Eng. Sci, 29, pp. 901-16, (1991). 